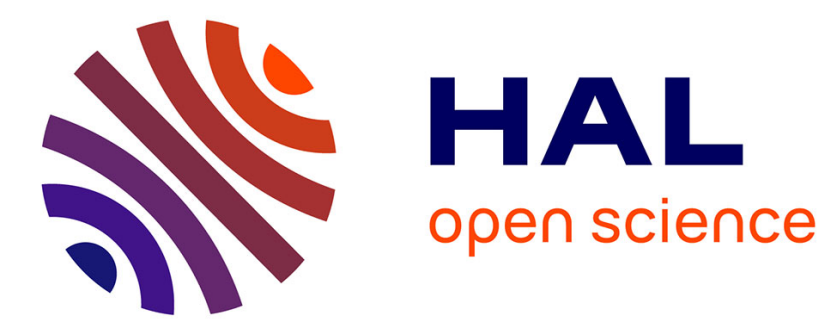

\title{
Benchmark 3D: CeVeFE-DDFV, a discrete duality scheme with cell/vertex/face+edge unknowns
}

\author{
Yves Coudière, Florence Hubert, Gianmarco Manzini
}

\section{To cite this version:}

Yves Coudière, Florence Hubert, Gianmarco Manzini. Benchmark 3D: CeVeFE-DDFV, a discrete duality scheme with cell/vertex/face+edge unknowns. Finite Volume for complex applications VI, Jun 2011, Praha, Czech Republic. pp.977-984. hal-00580558

\section{HAL Id: hal-00580558 https://hal.science/hal-00580558}

Submitted on 28 Mar 2011

HAL is a multi-disciplinary open access archive for the deposit and dissemination of scientific research documents, whether they are published or not. The documents may come from teaching and research institutions in France or abroad, or from public or private research centers.
L'archive ouverte pluridisciplinaire HAL, est destinée au dépôt et à la diffusion de documents scientifiques de niveau recherche, publiés ou non, émanant des établissements d'enseignement et de recherche français ou étrangers, des laboratoires publics ou privés. 


\section{Benchmark 3D: CeVeFE-DDFV, a discrete duality scheme with cell/vertex/face+edge unknowns}

Yves Coudière, Florence Hubert and Gianmarco Manzini

\section{Presentation of the scheme}

The method that we investigate in this contribution was proposed by Y. Coudière and F. Hubert in [1] as a three-dimensional (3D) extension of the finite volume scheme previously studied by F. Hermeline in [4] and K. Domelevo and P. Omnès in [3]. This method belongs to the family of Discrete Duality Finite Volume (DDFV) methods, which can naturally handle anisotropic or non-linear problems on general distorted meshes.

In this benchmark paper, we present the results obtained by using the formulation in [1] and the variant for discontinuous permeabilities that is presented in the proceeding paper [2].

The DDFV method that we consider herein makes use of three polyhedral meshes for the solution approximation, denoted by $\mathscr{M}, \mathscr{N}, \mathscr{F} \mathscr{E}$, and the mesh of diamonds for the solution gradient approximation, denoted by $\mathscr{D}$.

We denote the control volumes of the primal mesh $\mathscr{M}$ by $\mathrm{K}$ and $\mathrm{L}$, and with every primal cell we associate an internal point, e.g., $x_{\mathrm{K}} \in \mathrm{K}$. Different choices are possible, which give rise to different versions of the same scheme, such as the barycenters or the arithmetic average of the position vector of cell vertices (also called "isobarycenters"). For the results shown here, we used the second choice, but apparently there is no significant difference between the two choices mentioned above as far as accuracy and convergence behavior are concerned. The vertices, the edges, and the faces of mesh $\mathscr{M}$ are denoted by $x_{\mathrm{A}}, \mathrm{E}$ and F, respectively. Also, we denote the

Yves Coudière

Laboratoire de Mathématiques Jean Leray, Nantes, FRANCE, e-mail: Yves.Coudiere@

univ-nantes. fr

Florence Hubert

LATP, Université de Provence, Marseille, FRANCE, e-mail: fhubert@cmi • univ-mrs . fr

Gianmarco Manzini

IMATI and CESNA-IUSS, Pavia, ITALY, e-mail: gm.manzini@gmail.com 
midpoint of $\mathrm{E}$ by $x_{\mathrm{E}}$ and the barycenter of $\mathrm{F}$ by $x_{\mathrm{F}}$. We associate a degree of freedom (the scheme unknowns) with each one of these points; hence, the unknown scalar variable takes the form:

$$
u^{\mathscr{T}}=\left(\left(u_{\mathrm{K}}\right)_{K \in \mathscr{M}},\left(u_{\mathrm{A}}\right)_{A \in \mathscr{N}},\left(u_{\mathrm{E}}\right)_{E \in \mathscr{E}},\left(u_{\mathrm{F}}\right)_{F \in \mathscr{F}}\right) .
$$

We denote the collections of the boundary items (vertices, edges and faces) by $\partial \mathscr{N}$, $\partial \mathscr{F} \mathscr{E}$ and we introduce the set of boundary cells $\partial \mathscr{M}$ which is composed by the boundary faces here considered as degenerated control volumes. Dirichlet boundary conditions are easily introduced into the scheme through the set of boundary data

$$
\delta u^{\mathscr{T}}=\left(\left(u_{\mathrm{K}}\right)_{x_{\mathrm{K}} \in \partial \mathscr{M}},\left(u_{\mathrm{A}}\right)_{x_{\mathrm{A}} \in \partial \mathscr{N}},\left(u_{\mathrm{E}}\right)_{x_{\mathrm{E}} \in \partial \mathscr{F} \mathscr{E}},\left(u_{\mathrm{F}}\right)_{x_{\mathrm{F}} \in \partial \mathscr{F} \mathscr{E}}\right) .
$$

The scalar solution field $u$ is approximated by the degrees of freedom $\left(u^{\mathscr{T}}, \delta u^{\mathscr{T}}\right)$.

The gradient formula is given on each diamond cell $\mathrm{D} \in \mathscr{D}$, which is the convex hull of the points K, $\mathrm{L}, x_{\mathrm{A}}, x_{\mathrm{B}}, x_{\mathrm{F}}, x_{\mathrm{E}}$, by

$$
\nabla_{\delta u}^{\mathrm{D}} u^{\mathscr{T}}=\frac{1}{3|\mathrm{D}|}\left(\left(u_{\mathrm{L}}-u_{\mathrm{K}}\right) N_{\mathrm{KL}}+\left(u_{\mathrm{B}}-u_{\mathrm{A}}\right) N_{\mathrm{AB}}+\left(u_{\mathrm{F}}-u_{\mathrm{E}}\right) N_{\mathrm{EF}}\right)
$$

using the normal vectors $N_{\mathrm{KL}}=\frac{1}{2}\left(x_{\mathrm{B}}-x_{\mathrm{A}}\right) \times\left(x_{\mathrm{F}}-x_{\mathrm{E}}\right), N_{\mathrm{AB}}=\frac{1}{2}\left(x_{\mathrm{F}}-x_{\mathrm{E}}\right) \times\left(x_{\mathrm{L}}-\right.$ $\left.x_{\mathrm{K}}\right)$ and $N_{\mathrm{EF}}=\frac{1}{2}\left(x_{\mathrm{L}}-x_{\mathrm{K}}\right) \times\left(x_{\mathrm{B}}-x_{\mathrm{A}}\right)$. Gradient formula (1) allows us to define the numerical flux through each interface of the control volumes of the three meshes $\mathscr{M}$, $\mathscr{N}$ and $\mathscr{F} \mathscr{E}$. Let $\mathbf{Q}$ be the linear space of piecewise constant vector fields defined on the mesh of diamonds $\mathscr{D}$ and $X$ be the linear space of triples of piecewise constant scalar fields defined on the three meshes $\mathscr{M}, \mathscr{N}$ and $\mathscr{F} \mathscr{E}$. Three finite volume schemes are written by using a discrete divergence operator that maps each vector field in $\mathbf{Q}$ to a triple of scalar functions in $X$. Formally, we introduce the operator

$$
\operatorname{div}^{\mathscr{T}}: \xi=\left(\xi_{\mathrm{D}}\right)_{\mathrm{D} \in \mathscr{D}} \in \mathbf{Q} \mapsto\left(\operatorname{div}^{\mathscr{M}} \xi, \operatorname{div}^{\mathscr{N}} \xi, \operatorname{div}^{\mathscr{F} \mathscr{E}} \xi\right) \in X
$$

whose components

$$
\operatorname{div}^{\mathscr{M}} \xi=\left(\operatorname{div}_{\mathrm{K}} \xi\right)_{\mathrm{K}}, \operatorname{div}^{\mathscr{N}} \xi=\left(\operatorname{div}_{\mathrm{A}} \xi\right)_{\mathrm{A}} \text { and } \operatorname{div}^{\mathscr{F} \mathscr{E}} \xi=\left\{\left(\operatorname{div}_{\mathrm{E}} \xi\right)_{\mathrm{E}},\left(\operatorname{div}_{\mathrm{F}} \xi\right)_{\mathrm{F}}\right\}
$$

are given by

$$
\begin{aligned}
|\mathrm{K}| \operatorname{div}_{\mathrm{K}} \xi & =\sum_{\mathrm{D} \in \mathrm{D}_{\mathrm{K}}} \xi_{\mathrm{D}} \cdot N_{\mathrm{KL}}, \quad|\mathrm{A}| \operatorname{div}_{\mathrm{A}} \xi=\sum_{\mathrm{D} \in \mathrm{D}_{\mathrm{A}}} \xi_{\mathrm{D}} \cdot N_{\mathrm{AB}}, \\
|\mathrm{E}| \operatorname{div}_{\mathrm{E}} \xi & =\sum_{\mathrm{D} \in \mathrm{D}_{\mathrm{E}}} \xi_{\mathrm{D}} \cdot N_{\mathrm{EF}}, \quad|\mathrm{F}| \operatorname{div}_{\mathrm{F}} \xi=\sum_{\mathrm{D} \in \mathrm{D}_{\mathrm{F}}} \xi_{\mathrm{D}} \cdot\left(-N_{\mathrm{EF}}\right) .
\end{aligned}
$$

In the previous statements, the symbols $\mathrm{D}_{\mathrm{K}}, \mathrm{D}_{\mathrm{A}}, \mathrm{D}_{\mathrm{E}}, \mathrm{D}_{\mathrm{F}}$ refer to the diamond cells which overlap the cells labeled by the corresponding subscripted indices K, A, E, and $\mathrm{L}$.

Since each of the $\operatorname{div}_{\mathrm{C}} \xi$ approximates $\frac{1}{|\mathrm{C}|} \int_{\mathrm{C}} \operatorname{div} \xi$ (for $\mathrm{C}=\mathrm{K}, \mathrm{A}, \mathrm{E}, \mathrm{F}$ ), the right hand side of the discrete problem is given by the piecewise constant projection of 
the function $f$ onto the space $X, \pi^{\mathscr{T}} f=\left\{\left(f_{\mathrm{K}}\right)_{\mathrm{K} \in \mathscr{M}},\left(f_{\mathrm{A}}\right)_{\mathrm{A} \in \mathscr{N}},\left(f_{\mathrm{E}}, f_{\mathrm{F}}\right)_{\mathrm{E} \in \mathscr{E}, \mathrm{F} \in \mathscr{F}}\right\}$ with $f_{\mathrm{C}}=\frac{1}{|\mathrm{C}|} \int_{\mathrm{C}} f(x) d x$ for any cell $\mathrm{C}=\mathrm{K} \in \mathscr{M}$ or $\mathrm{A} \in \mathscr{N}$ or $\mathrm{F}$ or $\mathrm{E} \in \mathscr{F} \mathscr{E}$.

The CeVeFE-DDFV scheme reads:

$$
-\operatorname{div}^{\mathscr{T}}\left(\mathbf{K}_{\mathrm{D}} \nabla_{\delta u}^{\mathrm{D}} u^{\mathscr{T}}\right)=\pi^{\mathscr{T}} f,
$$

where $\mathbf{K}_{\mathrm{D}}=\frac{1}{|\mathrm{D}|} \int_{\mathrm{D}} \mathbf{K}(x) d x$ is a piecewise constant tensor field on the mesh of the diamond cells. The scheme in (4) originates a symmetric and positive-definite linear system of equations (see [1] for a thourough discussion of the other properties). The case of the discontinuous permeability tensor of test 5 deserves a special treatment that is thouroughly discussed in [2].

\section{Mesure on the error}

To put the discrete and the exact solutions "at the same level", we use the projection $\pi^{\mathscr{T}} u_{e}$ of the exact solution and the associated discrete gradient reconstruction $\nabla^{\mathscr{T}} \pi^{\mathscr{T}} u_{e}$. Approximation errors are evaluated through the following norms:

$$
\begin{aligned}
\text { erl2 }= & \left\|e^{\mathscr{T}}\right\|_{L^{2}} /\left\|\pi^{\mathscr{T}} u_{e}\right\|_{L^{2}} \text { with }\left\|e^{\mathscr{T}}\right\|_{L^{2}}^{2}=\frac{1}{3} \sum_{\mathrm{C} \in \mathscr{M} \cup \mathscr{N} \cup \mathscr{F} \mathscr{E}}\left|\mathrm{C} \| e_{\mathrm{C}}\right|^{2} \\
\text { ergrad }= & \left\|\nabla^{\mathscr{T}} e^{\mathscr{T}}\right\|_{L^{2}} /\left\|\nabla^{\mathscr{T}} \pi^{\mathscr{T}} u_{e}\right\|_{L^{2}} \text { with }\left\|\nabla^{\mathscr{T}} e^{\mathscr{T}}\right\|^{2}=\sum_{\mathrm{D} \in \mathscr{D}}\left|\mathrm{D} \| \nabla^{\mathrm{D}} e^{\mathscr{T}}\right|^{2} \\
\text { ener }= & \left(\mathbf{K}^{\mathscr{D}} \nabla^{\mathscr{T}} e^{\mathscr{T}}, \nabla^{\mathscr{T}} e^{\mathscr{T}}\right)_{L^{2}} /\left(\mathbf{K}^{\mathscr{D}} \nabla^{\mathscr{T}} \pi^{\mathscr{T}} u_{e}, \nabla^{\mathscr{T}} \pi^{\mathscr{T}} u_{e}\right)_{L^{2}} \\
& \text { with }\left(\mathbf{K}^{\mathscr{D}} \nabla^{\mathscr{T}} e^{\mathscr{T}}, \nabla^{\mathscr{T}} e^{\mathscr{T}}\right)_{L^{2}}=\sum_{\mathrm{D} \in \mathscr{D}}|\mathrm{D}|\left(\mathbf{K}_{\mathrm{D}} \nabla^{\mathrm{D}} e^{\mathscr{T}}, \nabla^{\mathrm{D}} e^{\mathscr{T}}\right)
\end{aligned}
$$

In the case of the discontinuous tensor of test 5 , the diamond cell $\mathrm{D}$ is divided in two subdiamond cells, namely, $\mathrm{D}_{\mathrm{K}}$ and $\mathrm{D}_{\mathrm{L}}$. The gradient $\nabla^{\mathrm{D}} u$ is constant on $\mathrm{D}_{\mathrm{K}}$ (respectively, $\mathrm{D}_{\mathrm{L}}$ ) with value $\nabla_{\mathrm{K}}^{\mathrm{D}} u$ (respectively, $\nabla_{\mathrm{L}}^{\mathrm{D}} u$ ). The quantities $\left\|\nabla^{\mathscr{T}} e^{\mathscr{T}}\right\|_{L^{2}}^{2}$ and $\left(\mathbf{K}^{\mathscr{D}} \nabla^{\mathscr{T}} e^{\mathscr{T}}, \nabla^{\mathscr{T}} e^{\mathscr{T}}\right)_{L^{2}}$ become

$$
\left\|\nabla^{\mathscr{T}} e^{\mathscr{T}}\right\|_{L^{2}}^{2}=\sum_{\mathrm{D} \in \mathscr{D}}\left(\left|\mathrm{D}_{\mathrm{K}}\right|\left|\nabla_{\mathrm{D}_{\mathrm{K}}} e^{\mathscr{T}}\right|^{2}+\left|\mathrm{D}_{\mathrm{L}}\right|\left|\nabla_{\mathrm{D}_{\mathrm{L}}} e^{\mathscr{T}}\right|^{2}\right)
$$

and

$$
\left(\mathbf{K}^{\mathscr{D}} \nabla^{\mathscr{T}} e^{\mathscr{T}}, \nabla^{\mathscr{T}} e^{\mathscr{T}}\right)_{L^{2}}=\sum_{\mathrm{D} \in \mathscr{D}}\left(\left|\mathrm{D}_{\mathrm{K}}\right|\left(\mathbf{K}_{\mathrm{D}_{\mathrm{K}}} \nabla_{\mathrm{D}_{\mathrm{K}}} e^{\mathscr{T}}, \nabla_{\mathrm{D}_{\mathrm{K}}} e^{\mathscr{T}}\right)+\left|\mathrm{D}_{\mathrm{L}}\right|\left(\mathbf{K}_{\mathrm{D}_{\mathrm{L}}} \nabla_{\mathrm{D}_{\mathrm{L}}} e^{\mathscr{T}}, \nabla_{\mathrm{D}_{\mathrm{L}}} e^{\mathscr{T}}\right)\right)
$$

\section{Numerical results}

The following results were obtained by using a BiCG-stab solver with ILU(0) preconditioner (routine MI26 of HSL implementation). 
- Test 1 Mild anisotropy, $u(x, y, z)=1+\sin (\pi x) \sin \left(\pi\left(y+\frac{1}{2}\right)\right) \sin \left(\pi\left(z+\frac{1}{3}\right)\right)$ $\min =0, \max =2$, Tetrahedral meshes

\begin{tabular}{|c|ccccccc|}
$\mathrm{i}$ & $\mathrm{nu}$ & nmat & umin & uemin & umax & uemax & normg \\
\hline 1 & 7777 & 100569 & $6.09 \mathrm{E}-03$ & $1.05 \mathrm{E}-02$ & 1.988 & 1.980 & 1.790 \\
2 & 15495 & 208527 & $7.48 \mathrm{E}-03$ & $9.35 \mathrm{E}-03$ & 1.995 & 1.994 & 1.793 \\
3 & 31139 & 431667 & $3.19 \mathrm{E}-03$ & $5.93 \mathrm{E}-03$ & 1.993 & 1.993 & 1.795 \\
4 & 62419 & 885735 & $1.48 \mathrm{E}-03$ & $2.98 \mathrm{E}-03$ & 1.996 & 1.996 & 1.796 \\
5 & 125993 & 1823199 & $1.56 \mathrm{E}-03$ & $2.28 \mathrm{E}-03$ & 2.000 & 1.999 & 1.797 \\
6 & 254657 & 3746829 & $1.93 \mathrm{E}-03$ & $2.70 \mathrm{E}-03$ & 1.999 & 1.998 & 1.798 \\
\hline
\end{tabular}

\begin{tabular}{|c|ccccccc|}
$\mathrm{i}$ & $\mathrm{nu}$ & erl2 & ratiol2 & ergrad & ratiograd & ener & ratioener \\
\hline 1 & 7777 & $0.228 \mathrm{E}-02$ & - & $0.562 \mathrm{E}-01$ & - & $0.528 \mathrm{E}-01$ & - \\
2 & 15495 & $0.147 \mathrm{E}-02$ & 1.904 & $0.441 \mathrm{E}-01$ & 1.051 & $0.415 \mathrm{E}-01$ & 1.054 \\
3 & 31139 & $0.916 \mathrm{E}-03$ & 2.036 & $0.349 \mathrm{E}-01$ & 1.011 & $0.327 \mathrm{E}-01$ & 1.021 \\
4 & 62419 & $0.573 \mathrm{E}-03$ & 2.025 & $0.276 \mathrm{E}-01$ & 1.006 & $0.258 \mathrm{E}-01$ & 1.022 \\
5 & 125993 & $0.374 \mathrm{E}-03$ & 1.819 & $0.219 \mathrm{E}-01$ & 0.994 & $0.206 \mathrm{E}-01$ & 0.969 \\
6 & 254657 & $0.231 \mathrm{E}-03$ & 2.067 & $0.174 \mathrm{E}-01$ & 0.983 & $0.163 \mathrm{E}-01$ & 0.990 \\
\hline
\end{tabular}

- Test 1 Mild anisotropy, $u(x, y, z)=1+\sin (\pi x) \sin \left(\pi\left(y+\frac{1}{2}\right)\right) \sin \left(\pi\left(z+\frac{1}{3}\right)\right)$ $\min =0, \max =2$, Voronoi meshes

\begin{tabular}{|c|ccccccc|}
$\mathrm{i}$ & $\mathrm{nu}$ & $\mathrm{nmat}$ & umin & uemin & umax uemax & normg \\
\hline 1 & 345 & 4559 & $7.93 \mathrm{E}-02$ & $1.51 \mathrm{E}-01$ & 1.875 & 1.844 & 1.719 \\
2 & 933 & 12811 & $4.79 \mathrm{E}-02$ & $4.74 \mathrm{E}-02$ & 1.989 & 1.982 & 1.785 \\
3 & 2075 & 29291 & $5.46 \mathrm{E}-02$ & $5.64 \mathrm{E}-02$ & 1.987 & 1.978 & 1.794 \\
4 & 3963 & 56947 & $3.25 \mathrm{E}-02$ & $3.23 \mathrm{E}-02$ & 2.000 & 1.996 & 1.795 \\
5 & 6909 & 101229 & $1.28 \mathrm{E}-02$ & $3.17 \mathrm{E}-02$ & 2.000 & 1.996 & 1.797 \\
\hline
\end{tabular}

\begin{tabular}{|c|ccccccc|}
$\mathrm{i}$ & nu & erl2 & ratiol2 & ergrad & ratiograd & ener & ratioener \\
\hline 1 & 345 & $0.274 \mathrm{E}-01$ & - & $0.179 \mathrm{E}+00$ & - & $0.162 \mathrm{E}+00$ & - \\
2 & 933 & $0.223 \mathrm{E}-01$ & 0.622 & $0.149 \mathrm{E}+00$ & 0.556 & $0.139 \mathrm{E}+00$ & 0.458 \\
3 & 2075 & $0.119 \mathrm{E}-01$ & 2.364 & $0.102 \mathrm{E}+00$ & 1.409 & $0.964 \mathrm{E}-01$ & 1.373 \\
4 & 3963 & $0.819 \mathrm{E}-02$ & 1.724 & $0.835 \mathrm{E}-01$ & 0.933 & $0.782 \mathrm{E}-01$ & 0.972 \\
5 & 6909 & $0.599 \mathrm{E}-02$ & 1.694 & $0.691 \mathrm{E}-01$ & 1.021 & $0.655 \mathrm{E}-01$ & 0.953 \\
\hline
\end{tabular}


- Test 1 Mild anisotropy, $u(x, y, z)=1+\sin (\pi x) \sin \left(\pi\left(y+\frac{1}{2}\right)\right) \sin \left(\pi\left(z+\frac{1}{3}\right)\right)$ $\min =0, \max =2$, Kershaw meshes

\begin{tabular}{|c|ccccccc|}
$\mathrm{i}$ & $\mathrm{nu}$ & $\mathrm{nmat}$ & umin & uemin & umax & uemax & normg \\
\hline 1 & 3375 & 49071 & $5.67 \mathrm{E}-02$ & $3.43 \mathrm{E}-02$ & 1.940 & 1.974 & 1.767 \\
2 & 29791 & 455895 & $9.19 \mathrm{E}-03$ & $7.33 \mathrm{E}-03$ & 1.988 & 1.991 & 1.782 \\
3 & 250047 & 3916359 & $2.42 \mathrm{E}-03$ & $1.59 \mathrm{E}-03$ & 1.999 & 1.998 & 1.793 \\
4 & 2048383 & 32446751 & $6.52 \mathrm{E}-04$ & $6.17 \mathrm{E}-04$ & 2.000 & 1.999 & 1.797 \\
\hline
\end{tabular}

\begin{tabular}{|c|ccccccc|}
$\mathrm{i}$ & $\mathrm{nu}$ & erl2 & ratiol2 & ergrad & ratiograd & ener & ratioener \\
\hline 1 & 3375 & $0.287 \mathrm{E}-01$ & - & $0.481 \mathrm{E}+00$ & - & $0.589 \mathrm{E}+00$ & - \\
2 & 29791 & $0.113 \mathrm{E}-01$ & 1.289 & $0.218 \mathrm{E}+00$ & 1.088 & $0.233 \mathrm{E}+00$ & 1.277 \\
3 & 250047 & $0.330 \mathrm{E}-02$ & 1.730 & $0.904 \mathrm{E}-01$ & 1.243 & $0.953 \mathrm{E}-01$ & 1.260 \\
4 & 2048383 & $0.859 \mathrm{E}-03$ & 1.922 & $0.395 \mathrm{E}-01$ & 1.180 & $0.422 \mathrm{E}-01$ & 1.161 \\
\hline
\end{tabular}

- Test 1 Mild anisotropy, $u(x, y, z)=1+\sin (\pi x) \sin \left(\pi\left(y+\frac{1}{2}\right)\right) \sin \left(\pi\left(z+\frac{1}{3}\right)\right)$ $\min =0, \max =2$, Checkerboard meshes

\begin{tabular}{|c|ccccccc}
$\mathrm{i}$ & $\mathrm{nu}$ & $\mathrm{nmat}$ & $\mathrm{umin}$ & uemin & umax & uemax & normg \\
\hline 1 & 239 & 2871 & $8.58 \mathrm{E}-02$ & $8.40 \mathrm{E}-02$ & 1.903 & 1.916 & 1.795 \\
2 & 2543 & 34927 & $2.90 \mathrm{E}-02$ & $2.13 \mathrm{E}-02$ & 1.971 & 1.979 & 1.804 \\
3 & 23135 & 336735 & $4.68 \mathrm{E}-03$ & $5.35 \mathrm{E}-03$ & 1.995 & 1.995 & 1.800 \\
4 & 196799 & 2943487 & $1.69 \mathrm{E}-03$ & $1.34 \mathrm{E}-03$ & 1.998 & 1.999 & 1.799 \\
5 & 1622399 & 24588351 & $2.88 \mathrm{E}-04$ & $3.35 \mathrm{E}-04$ & 2.000 & 2.000 & 1.799 \\
\hline
\end{tabular}

\begin{tabular}{|c|c|c|c|c|c|c|c|}
\hline & nu & erl2 & ratiol2 & ergrad & tiog & ener & $t e$ \\
\hline & 239 & 307E-01 & - & $0.141 \mathrm{E}+00$ & - & $0.139 \mathrm{E}+00$ & - \\
\hline & 2543 & 0 & 1.190 & & & & \\
\hline & 23135 & 2 & & & & & \\
\hline & 96799 & $0.830 \mathrm{E}-03$ & 1.905 & 0.29 & & 01 & 0.9 \\
\hline & 239 & $210 \mathrm{E}-03$ & 1.955 & $0.154 \mathrm{E}-01$ & 0.937 & $0.147 \mathrm{E}-01$ & 0.945 \\
\hline
\end{tabular}

- Test 2 Heterogeneous anisotropy, $u(x, y, z)=x^{3} y^{2} z+x \sin (2 \pi x z) \sin (2 \pi x y) \sin (2 \pi z), \min =-0.862, \max =1.0487$, Prism meshes

\begin{tabular}{|c|ccccccc|}
$\mathrm{i}$ & $\mathrm{nu}$ & $\mathrm{nmat}$ & umin & uemin & umax & uemax & normg \\
\hline 1 & 12179 & 188089 & $-8.55 \mathrm{E}-01$ & $-8.46 \mathrm{E}-01$ & 1.014 & 1.009 & 1.693 \\
2 & 96759 & 1545215 & $-8.55 \mathrm{E}-01$ & $-8.57 \mathrm{E}-01$ & 1.026 & 1.031 & 1.706 \\
3 & 325739 & 5259545 & $-8.61 \mathrm{E}-01$ & $-8.59 \mathrm{E}-01$ & 1.037 & 1.035 & 1.708 \\
4 & 771119 & 12518433 & $-8.60 \mathrm{E}-01$ & $-8.60 \mathrm{E}-01$ & 1.040 & 1.041 & 1.709 \\
\hline
\end{tabular}




\begin{tabular}{|c|ccccccc|}
$\mathrm{i}$ & $\mathrm{nu}$ & erl2 & ratiol2 & ergrad & ratiograd & ener & ratioener \\
\hline 1 & 12179 & $0.392 \mathrm{E}-01$ & - & $0.811 \mathrm{E}-01$ & - & $0.803 \mathrm{E}-01$ & - \\
2 & 96759 & $0.109 \mathrm{E}-01$ & 1.854 & $0.392 \mathrm{E}-01$ & 1.054 & $0.397 \mathrm{E}-01$ & 1.019 \\
3 & 325739 & $0.502 \mathrm{E}-02$ & 1.917 & $0.256 \mathrm{E}-01$ & 1.051 & $0.261 \mathrm{E}-01$ & 1.040 \\
4 & 771119 & $0.287 \mathrm{E}-02$ & 1.942 & $0.190 \mathrm{E}-01$ & 1.039 & $0.194 \mathrm{E}-01$ & 1.034 \\
\hline
\end{tabular}

- Test 3 Flow on random meshes, $u(x, y, z)=\sin (\pi x) \sin (\pi y) \sin (\pi z)$, $\min =0, \max =1$, Random meshes

\begin{tabular}{|c|ccccccc|}
$\mathrm{i}$ & $\mathrm{nu}$ & $\mathrm{nmat}$ & $\mathrm{umin}$ & uemin & umax & uemax & normg \\
\hline 1 & 343 & 4447 & $-4.25 \mathrm{E}+01$ & $-9.78 \mathrm{E}-01$ & 49.169 & 0.931 & 38.139 \\
2 & 3375 & 49855 & $-2.22 \mathrm{E}+01$ & $-9.94 \mathrm{E}-01$ & 21.970 & 0.982 & 21.514 \\
3 & 29791 & 466111 & $-6.96 \mathrm{E}+00$ & $-9.95 \mathrm{E}-01$ & 7.051 & 0.993 & 12.536 \\
4 & 250047 & 4019647 & $-2.67 \mathrm{E}+00$ & $-9.98 \mathrm{E}-01$ & 2.725 & 0.998 & 7.541 \\
\hline
\end{tabular}

\begin{tabular}{|c|ccccccc|}
$\mathrm{i}$ & $\mathrm{nu}$ & erl2 & ratiol2 & ergrad & ratiograd & ener & ratioener \\
\hline 1 & 343 & $0.147 \mathrm{E}+03$ & - & $0.238 \mathrm{E}+02$ & - & $0.162 \mathrm{E}+01$ & - \\
2 & 3375 & $0.956 \mathrm{E}+01$ & 3.589 & $0.121 \mathrm{E}+02$ & 0.892 & $0.888 \mathrm{E}+00$ & 0.787 \\
3 & 29791 & $0.681 \mathrm{E}+00$ & 3.640 & $0.632 \mathrm{E}+01$ & 0.891 & $0.459 \mathrm{E}+00$ & 0.909 \\
4 & 250047 & $0.447 \mathrm{E}-01$ & 3.840 & $0.314 \mathrm{E}+01$ & 0.988 & $0.229 \mathrm{E}+00$ & 0.979 \\
\hline
\end{tabular}

- Test 4 Flow around a well, Well meshes, $\min =0, \max =5.415$

\begin{tabular}{|c|ccccccc|}
$\mathrm{i}$ & $\mathrm{nu}$ & nmat & umin & uemin & umax & uemax & normg \\
\hline 1 & 5868 & 86728 & $3.83 \mathrm{E}-01$ & $4.13 \mathrm{E}-01$ & 5.317 & 5.317 & 1596.292 \\
2 & 15776 & 243104 & $2.37 \mathrm{E}-01$ & $2.43 \mathrm{E}-01$ & 5.328 & 5.328 & 1611.158 \\
3 & 36846 & 580244 & $1.54 \mathrm{E}-01$ & $1.54 \mathrm{E}-01$ & 5.329 & 5.329 & 1617.452 \\
4 & 84546 & 1350382 & $1.17 \mathrm{E}-01$ & $1.18 \mathrm{E}-01$ & 5.330 & 5.330 & 1620.143 \\
5 & 177590 & 2860258 & $8.96 \mathrm{E}-02$ & $8.98 \mathrm{E}-02$ & 5.339 & 5.339 & 1621.406 \\
6 & 329236 & 5329338 & $7.22 \mathrm{E}-02$ & $7.23 \mathrm{E}-02$ & 5.345 & 5.345 & 1622.053 \\
7 & 580190 & 9422104 & $5.66 \mathrm{E}-02$ & $5.64 \mathrm{E}-02$ & 5.361 & 5.361 & 1622.472 \\
\hline
\end{tabular}

\begin{tabular}{|c|ccccccc|}
$\mathrm{i}$ & $\mathrm{nu}$ & erl2 & ratiol2 & ergrad & ratiograd & ener & ratioener \\
\hline 1 & 5868 & $0.141 \mathrm{E}-04$ & - & $0.128 \mathrm{E}+00$ & - & $0.116 \mathrm{E}+00$ & - \\
2 & 15776 & $0.476 \mathrm{E}-05$ & 3.290 & $0.877 \mathrm{E}-01$ & 1.144 & $0.781 \mathrm{E}-01$ & 1.212 \\
3 & 36846 & $0.208 \mathrm{E}-05$ & 2.924 & $0.610 \mathrm{E}-01$ & 1.283 & $0.542 \mathrm{E}-01$ & 1.287 \\
4 & 84546 & $0.141 \mathrm{E}-05$ & 1.411 & $0.466 \mathrm{E}-01$ & 0.975 & $0.408 \mathrm{E}-01$ & 1.033 \\
5 & 177590 & $0.914 \mathrm{E}-06$ & 1.747 & $0.362 \mathrm{E}-01$ & 1.021 & $0.316 \mathrm{E}-01$ & 1.023 \\
6 & 329236 & $0.609 \mathrm{E}-06$ & 1.976 & $0.293 \mathrm{E}-01$ & 1.026 & $0.258 \mathrm{E}-01$ & 0.998 \\
7 & 580190 & $0.422 \mathrm{E}-06$ & 1.941 & $0.244 \mathrm{E}-01$ & 0.964 & $0.214 \mathrm{E}-01$ & 0.971 \\
\hline
\end{tabular}


- Test 5 Discontinuous permeability, $u(x, y, z)=\sin (\pi x) \sin (\pi y) \sin (\pi z)$, $\min =0, \max =1$, Locally refined meshes

\begin{tabular}{|c|ccccccc|}
$\mathrm{i}$ & $\mathrm{nu}$ & $\mathrm{nmat}$ & umin & uemin & umax & uemax & normg \\
\hline 1 & 131 & 1017 & $-6.34 \mathrm{E}+01$ & $-1.00 \mathrm{E}+02$ & 64.462 & 100.000 & 85.763 \\
\hline 2 & 1215 & 8303 & $-3.10 \mathrm{E}+02$ & $-1.00 \mathrm{E}+02$ & 309.886 & 100.000 & 192.379 \\
\hline 3 & 10463 & 65007 & $-1.34 \mathrm{E}+02$ & $-1.00 \mathrm{E}+02$ & 134.323 & 100.000 & 139.345 \\
\hline 4 & 86847 & 509567 & $-1.09 \mathrm{E}+02$ & $-1.00 \mathrm{E}+02$ & 109.373 & 100.000 & 114.251 \\
\hline 5 & 707711 & 4024287 & $-1.02 \mathrm{E}+02$ & $-1.00 \mathrm{E}+02$ & 102.394 & 100.000 & 104.279 \\
\hline
\end{tabular}

\begin{tabular}{|c|ccccccc|}
$\mathrm{i}$ & $\mathrm{nu}$ & erl2 & ratiol2 & ergrad & ratiograd & ener & ratioener \\
\hline 1 & 131 & $0.218 \mathrm{E}+01$ & - & $0.450 \mathrm{E}+00$ & - & $0.406 \mathrm{E}+00$ & - \\
\hline 2 & 1215 & $0.193 \mathrm{E}+01$ & 0.159 & $0.187 \mathrm{E}+01$ & -1.917 & $0.623 \mathrm{E}+00$ & -0.578 \\
\hline 3 & 10463 & $0.862 \mathrm{E}-01$ & 4.334 & $0.828 \mathrm{E}+00$ & 1.134 & $0.297 \mathrm{E}+00$ & 1.033 \\
\hline 4 & 86847 & $0.517 \mathrm{E}-02$ & 3.989 & $0.407 \mathrm{E}+00$ & 1.006 & $0.147 \mathrm{E}+00$ & 0.995 \\
\hline 5 & 707711 & $0.326 \mathrm{E}-03$ & 3.953 & $0.203 \mathrm{E}+00$ & 0.994 & $0.734 \mathrm{E}-01$ & 0.994 \\
\hline
\end{tabular}

\section{Comments}

This finite volume method assigns one degree of freedom to any mesh item (cells, faces, edges, and vertices). For this reason, the scheme has a large number of degrees of freedom if compared to other finite volume methods or similar discretization techniques (such as mimetic finite differences). Nonetheless, the method was proved very effective both for two and three dimensional problems with strong anisotropic coefficients and using meshes with strongly distorted cells. Among the other advantages offered by the method, we mention the coercivity of the method that eases the convergence analysis and the fact that this finite volume method generally shows second order of accuracy in all numerical experiments where the exact solution is sufficiently regular. The results shown in the tables of the previous section confirm this general behavior.

All linear systems were solved efficiently by standard preconditioned Krylov methods as BiCG-stab or GMRES. Direct solvers for general asymmetric systems (UMFPACK) can also be used, but they normally require a huge memory storage, in particular for the biggest problems. In Table 1-2, we see an example of the performance of the different solvers offered by the benchmark site when solving Test 1 on the checkerboard meshes $8 \times 8 \times 8$ and $16 \times 16 \times 16$. The comparison reveals that PETSc implementation of the CG solver is the fastest one, in particular, when combined with the diagonal preconditioner (Jacobi). A good performance in terms of CPU costs is also provided by the ISTL-BiCGstab implementation using Jacobi or ILU(0) preconditioners. CPU times are usually smaller than those obtained by using the direct solver UMFPACK, which is also available in the benchmark site. 
For example, in the case of $8 \times 8 \times 8$-size mesh we note that UMFPACK requires a CPU time of 3.180 seconds.

\begin{tabular}{rrrrr} 
solver & precond & CPU time & \# iters & Rel. resid. \\
\hline PETSc-CG & Jacobi & 0.209 & 202 & $6.368 \mathrm{e}-11$ \\
PETSc-CG & none & 0.243 & 242 & $1.715 \mathrm{e}-10$ \\
ISTL-BiCGstab & ILU(0) & 0.404 & 53 & $6.832 \mathrm{e}-11$ \\
ISTL-BiCGstab & none & 0.563 & 167 & $3.656 \mathrm{e}-11$ \\
ISTL-BiCGstab & Jacobi & 0.680 & 120 & $4.415 \mathrm{e}-11$ \\
ISTL-GMRES & ILU(0) & 0.683 & 152 & $4.241 \mathrm{e}-11$ \\
\hline
\end{tabular}

Table $1 \mathrm{CeVeFe-DDFV}$ method, test 1 using checkerboard mesh, grid resolution $8 \times 8 \times 8$; CPU times are measured in seconds.

\begin{tabular}{rrrrr} 
solver & precond & CPU time & \# iters & Rel. resid. \\
\hline PETSc-CG & Jacobi & 3.946 & 369 & $4.248 \mathrm{e}-11$ \\
PETSc-CG & none & 4.989 & 471 & $8.038 \mathrm{e}-11$ \\
ISTL-CG & ILU(0) & 5.540 & 166 & $4.041 \mathrm{e}-11$ \\
ISTL-CG & none & 7.319 & 471 & $8.038 \mathrm{e}-11$ \\
ISTL-BiCGstab & ILU(0) & 8.681 & 107 & $3.873 \mathrm{e}-11$ \\
ISTL-CG & Jacobi & 10.989 & 368 & $4.281 \mathrm{e}-11$ \\
\hline
\end{tabular}

Table $2 \mathrm{CeVeFe-DDFV}$ method, test 1 using checkerboard mesh, grid resolution $16 \times 16 \times 16$; CPU times are measured in seconds.

\section{Aknowledgements}

The work of the first and second author was supported by Groupement de Recherche MOMAS. The work of the third author was partially supported by the Italian MIUR through the program PRIN2008.

\section{References}

1. Y. Coudière and F. Hubert. A 3D discrete duality finite volume method for nonlinear elliptic equation. Preprint HAL, URL: http://hal.archives-ouvertes.fr/hal-00456837/fr, 2010.

2. Y. Coudière, F. Hubert, and G. Manzini. A cevefe-ddfv scheme for discontinuous permeability tensors. In Finite Volume For Complex Applications, Problems And Perspectives. 6th International Conference (this volume), 2011.

3. K. Domelevo and P. Omnès. A finite volume method for the laplace equation on almost arbitrary two-dimensional grids. M2AN, Math. Model. Numer. Anal., 39(6):1203-1249, 2005.

4. F. Hermeline. Approximation of diffusion operators with discontinuous tensor coefficients on distorted meshes. Comput. Methods Appl. Mech. Engrg., 192(16):1939-1959, 2003.

The paper is in final form and no similar paper has been or is being submitted elsewhere. 